\title{
DE LA CONSOLIDACIÓN A LA CESANTÍA: EVOLUCIÓN DEL PROFESORADO DE MEDICINA EN LA UNIVERSIDAD LITERARIA DE SEVILLA (1833-1845)
}

\author{
Juan L. Carrillo \\ Departamento de Ciencas Socio-Sanitarias \\ Facultad de Medicina, Sevilla
}

\section{RESUMEN}

En el presente artículo se analiza la evolución del profesorado de medicina en la Universidad Literaria de Sevilla entre 1833 y 1845, fecha esta última en la que se procedió a la supresión de los estudios médicos en la ciudad de Sevilla. En 1834 se produjo una consolidación del profesorado por medio de una oposiciones descentralizadas; en 1843, ahora por nombramiento gubernamental, se estructuró un profesorado destinado a impartir sus enseñanzas en el recién creado Colegio de Prácticos en el Arte de Curar que se reclutó básicamente del antiguo profesorado de la Facultad de Medicina suprimida y en 1845 todo él quedó cesante, analizándose las diferentes salidas profesionales. Se toman muy en cuenta las condiciones locales que posibilitaron la creación y efímera existencia de dos Cátedras —una de Cirugía y otra de Obstetricia— entre 1840 y 1842.

PALABRAS CLAVE: universidad, Sevilla, siglo XIX, cirugía, medicina.

\section{SUMMARY}

This article analyses the evolution of the professors of medicine at the Literary University of Seville between 1833 and 1845, the latter being the year in which medical studies were eliminated from the city of Seville. The consolidation of the medical teaching staff took place in 1834 thanks to some decentralised public examinations. In 1843 and now due to a government appointment, the medical faculty was assigned to teach in the newly created «Colegio de Prácticos en el Arte de Curar» (School of Practitioners in the Art of Healing) which recruited its members from the professors of the former Faculty of Medicine, the one that had been eliminated. As the members of this institution were suspended in 1845 , there is an analysis of how they could be relocated as far as their professional career was concerned. The local conditions that favoured the creation and the ephemeral existence (between 1840 and 1842) of two professorships- one in Surgery and the other in Obstetrics-are very seriously taken into consideration.

KEY WORDS: university, Sevilla, $19^{\text {th }}$ century, surgery, medicine. 
En estos últimos años se ha producido un renovado interés por los estudios históricos relativos a la enseñanza de la medicina en la España de los siglos XIX y XX. No son ajenos al mismo los problemas derivados de la reforma de dichas enseñanzas en nuestro país (autonomía universitaria, política en materia de profesorado, planes de estudio, enseñanza clínica, etc); situación ésta que requiere disponer de referentes históricos que clarifiquen los orígenes de la problemática actual. Los trabajos publicados por José Luis Peset entre 1968 y 1974 han sido —y aun lo son- el referente más válido del que disponíamos al proporcionarnos una visión amplia y coherente, especialmente del siglo XIX.

Dos acontecimientos han venido a dinamizar los estudios sobre la enseñanza de la medicina. Por una parte la publicación por la Fundación Uriach 1838 de dos volúmenes coordinados por José Danón bajo el título La enseñanza de la Medicina en la Universidad Española (Barcelona, 1998-2001) y que recogen la historia de la enseñanza en los dos últimos siglos. Por otra la Ponencia «Historia de la enseñanza de la medicina en España» desarrollada en el XI Congreso Nacional de Historia de la Medicina (Santiago de Compostela, 1998) a la que se presentaron un total de 21 comunicaciones, de las cuales sólo una no correspondía a los siglos XIX y XX. El resultado de ambos acontecimientos debe servir de estímulo para proseguir la tarea emprendida y a ello responde en presente artículo.

\section{I) LA CONSOLIDACIÓN DEL PROFESORADO EN LA FACULTAD DE MEDICINA DE SEVI-} LLA: LAS OPOSICIONES DE 1834

En los primeros meses de 1834 la Universidad Literaria de Sevilla declaró vacantes las cuatro Cátedras de Instituciones Médicas, poniéndose de inmediato en marcha un proceso administrativo y académico tendente a su ocupación en propiedad ${ }^{1}$. Este proceso se ejecutó bajo la normativa establecida en los artículos 205 y 206 del «Nuevo Plan general de Estudios» de $1824^{2}$ y concluyó un año después cuando se les dio posesión a los últimos ganadores de las oposiciones. Siguiendo el orden de celebración de las mismas, fueron éstos: la Cátedra de Instituciones de $4^{\circ}$ año (Patología Especial y Nosografía Médica) fue obtenida por Serafín Adame de Vargas Jiménez

1 Archivo Histórico Universitario de Sevilla (AHUS). Cátedras. Oposiciones, libro 928, fol. 14 [Auto del Rector Francisco de Paula Cerero de 17 de enero de 1834]. Este libro tiene doble paginación independiente. Para una aproximación a la historia de la enseñanza de la medicina en Sevilla entre 1824 y 1845, cf. NAVArro Hinojosa, R. (1991), La Universidad de Sevilla de 1824 a 1845: Organización y Curriculum, Sevilla, Secretariado de Publicaciones de la Universidad de Sevilla, pp. 441-495.

2 Real Cédula de S. M. y Señores del Consejo por la cual se manda observar en todas las Universidades y demás establecimientos del Reino el Nuevo Plan general de Estudios inserto en ella. Madrid, Imprenta Real, 1825 [Real Orden de 14 de octubre de 1824]. 
(c.1774-1852); la de Instituciones Médicas de $3^{\circ}$ año (Terapéutica, Materia Médica y Medicina Legal) por Joaquín Sánchez-Reciente Díaz (c.1801-d.1844); Instituciones de $2^{\circ}$ año (Fisiología, Patología e Higiene) por Manuel Campos Vallejo (c.17801848) y la de Anatomía por Fernando Velasco Libanto (c.1800-1836) 3 .

Este proceso presenta varios niveles de desajustes que son necesarios poner en evidencia para poder entender más cabalmente la situación de la Universidad Española y en particular la de sus Facultades de Medicina. El primer nivel de desajuste es el que se produce entre el gran despliegue informativo y la baja concurrencia. El Rector de la Universidad venía obligado a hacer pública la convocatoria en todas las Universidades del país, institución que hipotéticamente debería aportar candidatos. Junto a esta difusión, el propio contenido del Edicto de convocatoria debía suministrar información sobre las características y condiciones para acceder a las plazas en disputa (renta, titulación y grado académico requerido, etc.) ${ }^{4}$. A pesar de hacerse de tal modo en la Universidad de Sevilla, llama la atención el bajo nivel de concurrencia a las oposiciones: para las Cátedras de Anatomía e Instituciones de $3^{\circ}$ y de $4^{\circ}$ año sólo hubo un candidato respectivamente, que procedía de la propia Universidad hispalense; para la de Instituciones $2^{\circ}$ año fueron dos, uno de ellos, el perdedor, sin vinculación a Universidad alguna y el otro también de la hispalense.

El segundo nivel de desajuste tiene que ver con las características de los propios concurrentes; entre los que podríamos calificar de opositores reales - aquellos que presentaban su candidatura a la Cátedra - y los «contrincantes virtuales», que se utilizaban cuando no existía suficiente número de opositores reales y con la finalidad de poder organizar las trincas ${ }^{5}$. Naturalmente los primeros nunca opositaban «por malicia, ni con ánimo de perjudicar a tercero sino solo para que se administre justicia», según la fórmula establecida ${ }^{6}$. El objetivo de un «virtual» era ser aprobado por los Censores y usar esto como mérito en su carrera académica y profesional. En el caso de las oposiciones a que nos venimos refiriendo fue necesario recurrir a ellos

\footnotetext{
3 AHUS. Cátedras. Provisiones, libro 933, fols. 18-34.

4 En este caso la renta era de 5.000 rls; haber alcanzado el Grado de Bachiller en Medicina y cursado los dos años de Clínica; para tener derecho a jubilación alcanzar el Grado de Licenciado en Medicina en el plazo de seis meses y, por otra parte, debían presentar certificaciones de buena conducta. Reproducimos lo más sustancial del certificado que se le expidió a Campos Vallejo en fecha 22 de septiembre de 1834: «ha manifestado los sentimientos más religiosos y puros, inspirando a sus discípulos doctrinas sanas y adhesión al Gobierno monárquico». AHUS. Cátedras. Oposiciones, libro 929, fol. 255.

5 Estos opositores actuaban solamente en el primer y segundo ejercicio. El tercero o «examen secreto o privado» consistente en responder a preguntas de los Censores sobre cualquier parte de la medicina y ramas auxiliares, sólo era ejecutado por el opositor real. Del mismo modo ocurría con el ejercicio teóricopráctico de Anatomía, entre el segundo y el secreto, en las oposiciones a la Cátedra de esta disciplina.

6 AHUS. Cátedras. Oposiciones, libro 928, fol. 22 [Solicitud de Adame de fecha 25 de febrero de 1834]
} 
para las de Instituciones de $3^{\circ}$ y de $4^{\circ}$ año ${ }^{7}$; no ocurrió así para las dos restantes ya que la Inspección General de Instrucción Pública mandató que ambas se ejecutasen bajo la misma convocatoria y ejercicios ${ }^{8}$. Así pues los dos opositores reales aspirantes a la Cátedra de Instituciones de $2^{\circ}$ año - Manuel Campos Vallejo y Juan Nepomuceno Díaz Álvarez (n. 1803) - actuaron como «contrincantes» del candidato de la de Anatomía y éste - Fernando Velasco - en la de aquéllos. Ante tal circunstancia se recurrió a utilizar para las dos primeras Cátedras mencionadas a los sustitutos de Cátedras de la propia Universidad: para la primera a Joaquín Ballesteros Sousa (1804-1877) y a Joaquín Ma Doy Burgos (1811-1878), sustitutos respectivamente de Instituciones de $2^{\circ}$ año y de Anatomía y para la segunda a Manuel Pérez Martínez (c.1802-d.1865), sustituto de esa misma Cátedra y a Francisco Porrúa Velázquez (1808-d.1856) que lo era de Clínica9. En definitiva los que debían oponerse al candidato real eran personas que ocupaban un puesto docente en la Universidad por propuesta al Claustro de los propios interesados.

El tercer nivel de desajuste está representado por el alto grado de afinidad entre opositores y Censores al pertenecer todos al Claustro de Catedráticos de la Facultad de Medicina. En tal sentido hay que entender al Claustro de cualquier Facultad Universitaria, en este caso el de Medicina, como una potente estructura de poder académico, imposible de superar por cualquier candidato foráneo. Posiblemente éste fuera un factor disuasorio para ellos. Del Claustro de Medicina salieron los Censores que acompañaron al Rector en el desarrollo de la oposición; realizada ésta correspondía a la Inspección General de Instrucción Pública examinar el expediente enviado por la Universidad convocante y elevarlo, en esta coyuntura política, a la Reina Gobernadora. Por otra parte el Claustro de Medicina - al que pertenecían los opositores locales- establecía un «pool» de 200 títulos o capítulos de la asignatura de los que se sortearían tres y el opositor elegiría uno para presentar a debate sus proposiciones. En esto consistía el primer ejercicio de la oposición. Igualmente elegía los libros que servirían para determinar la lección que «de repente y en castellano», debía desarrollar el opositor en el segundo ejercicio ${ }^{10}$.

Actuaron como Censores, a propuesta de siete miembros del Claustro General elegidos mediante sorteo, los siguiente miembros del Claustro de Medicina: para la oposición de Adame, Francisco Velázquez Reyes (1779-d. 1840) Catedrático sustitu-

7 AHUS. Cátedras. Oposiciones, libro 928, fol. 36; libro 929, fol. 150.

8 AHUS. Cátedras. Oposiciones, libro 929, fol. 242 [Oficio de la Inspección General de Instrucción Pública de 22 de julio de 1834].

9 AHUS. Cátedras. Oposiciones, libro 928, fol. 36; libro 929, fol. 150.

10 El desarrollo de las tres oposiciones puede seguirse en los correspondientes expedientes: muy completo el de las Cátedras de Anatomía e Instituciones de $2^{\circ}$ año, así como el de Instituciones de $4^{\circ}$ año; incompleto y desordenado el de Instituciones de $2^{\circ}$ año. AHUS. Cátedras. Oposiciones, libro 928, fols. 14-42; libro 929 , fols. $150-155$ y fols. $240-265 \mathrm{v}$. 
to de Clínica, Campos Vallejo, en ese momento sustituto de Instituciones $2^{\circ}$ y Sánchez-Reciente en igual situación en Instituciones $3^{\circ}$; para la de Sánchez-Reciente actuaron Adame y Velázquez y Campos nuevamente y para las de Campos y Velasco, fueron Adame y Velázquez, de nuevo, y Sánchez-Reciente. Si reordenamos esta información veremos que Velázquez actuó de Censor en las tres oposiciones celebradas y que Adame, Campos y Sánchez-Reciente fueron Censores en dos ocasiones, cuando no eran candidatos. Por todo ello no llama la atención la airada protesta de Juan Nepomuceno Díaz Álvarez -independientemente de lo justificada o injustificada que estuviera en virtud de su cualificación científica y académica - dirigida al Rector impugnando el sistema de provisión de Cátedras Universitarias con un argumento muy consistente: jueces y opositores eran las mismas personas. Dos de los que apoyaron a Campos —Adame y Sánchez-Reciente - le eran deudores, al menos, desde las oposiciones celebradas anteriormente ${ }^{11}$.

El perfil sociológico de los cuatro nuevos propietarios de las Cátedras aclara considerablemente el alto nivel de provincialismo y endogamia universitaria en Sevilla durante el periodo anterior a las grandes reformas de la década de los cuarenta ${ }^{12}$. Todos eran sevillanos ${ }^{13}$, con una edad media en el momento de celebrarse la oposiciones de $45^{\prime} 2$ años ${ }^{14}$; dos de ellos eran hijos de médicos y uno de los dos hijo de Catedrático de Medicina ${ }^{15}$; todos habían estudiado en la Facultad de Medicina de la Universidad de Sevilla en la que habían alcanzado el Grado de Doctor, por cuya condición eran miembros del Claustro Universitario ${ }^{16}$; los cuatro habían desarrollado una tarea previa en esta Universidad como Catedráticos sustitutos ${ }^{17}$; todos ocupaban la Cátedra a la que opositaron ${ }^{18}$ y todos habían alcanzado un alto grado de reconoci-

11 AHUS. Cátedras. Oposiciones, libro 929, fols. 264-264v [Escrito de Juan Nepomuceno Díaz Álvarez al Rector de fecha 13 de diciembre de 1834].

12 Para establecer estos perfiles nos hemos valido fundamentalmente de la documentación siguiente: AHUS. Índice de carreras, libro 781, fols. 464-468; libro 795, fols. 500 (20)- 508; libro 848, fols. 381 402v y libro 843, fols. 555-578.

13 Habían nacido en la ciudad de Sevilla, menos Sánchez-Reciente que nació en Cantillana (Sevilla).

14 El de mayor edad era Adame con 60 años y el más joven Sánchez-Reciente con 33. Campos tenía 54 y Velasco 34

15 Adame era hijo de Andrés Adame de Vargas que había sido Catedrático de Anatomía y más tarde de Vísperas en Sevilla. El padre de Sánchez-Reciente era Bachiller en Medicina. No tenemos información de Campos ni de Velasco.

16 Adame estudió entre 1789 y 1793; Campos entre 1795 y 1799 ; Velasco entre 1815 y 1819 (los cursos $5^{\circ}$ y $6^{\circ}$ de Clínica entre 1819 y 1821) y Sánchez-Reciente entre 1816 y 1820 (la Clínica entre 1820 y 1822).

17 El que más antigüedad y continuidad tenía como sustituto era Adame (1803); Campos lo fue de forma intermitente desde 1810; Sánchez-Reciente desde 1822 y Velasco desde 1826.

18 Adame ocupaba Instituciones de $4^{\circ}$ año (vísperas) desde 1819; Campos se encargaba de Instituciones de $2^{\circ}$ año desde 1832 y Anatomía e Instituciones de $3^{\circ}$ año la ocupaban respectivamente Velasco y Sánchez-Reciente desde el mismo año de las oposiciones (1834) 
miento social en Sevilla ${ }^{19}$. De una cosa no hay la menor duda: el Claustro de Medicina surgido de las oposiciones de 1834 se caracterizó por su invisibilidad científica y académica, no traspasando los estrechos límites de su Sevilla natal.

\section{II) LA CRISIS DE 1836: EL «ARREGLO PROVISIONAL» Y SU APLICACIÓN EN LA FACUL- TAD DE MEDICINA DE SEVILLA}

Independientemente de la consideración científica y académica que puedan merecer los cuatro catedráticos de Instituciones Médicas de la Universidad de Sevilla, no cabe duda que el año de 1834 se utilizó para consolidar un profesorado que con mayor o menor acierto venía impartiendo sus enseñanzas, sin que el sistema permitiera alternativas a lo existente. Este proceso hay que incardinarlo en el marco de una línea seguida en la Universidad: si en 1830 se cubrió sólo una Cátedra en virtud de oposición y en 1831 y 1832 fueron dos respectivamente, en 1834 fueron seis y en 1835 se alcanzaron siete ${ }^{20}$. A partir de este momento se produce una paralización como consecuencia de la aplicación de la Real Orden de 1 de julio de 1835 por la que se suspendían las oposiciones a Cátedras de Universidad hasta que por el Gobierno se elaborase una reforma de los estudios.

Esta reforma no llegó hasta la década de los cuarenta, a pesar de existir una muy seria iniciativa política y jurídica en tal sentido. El 4 de agosto de 1836 la Reina Gobernadora sancionaba mediente Real Decreto una significativa reforma de la enseñanza, que en lo tocante a la Universitaria era poco reglamentista y algo inespecífi$\mathrm{ca}^{21}$. Los sucesos de La Granja del 12 de agosto y el hecho de tratarse de un Decreto no aprobado por las Cortes, condujeron a su no implantación y derogación ${ }^{22}$. Este

19 Adame, Campos y Sánchez-Reciente eran Académicos de la Real Academia de Medicina de Sevilla (antes Regia Sociedad de Medicina), no así Velasco. Los dos primeros presentaron un buen número de Memorias en dicha institución a lo largo de las dos primeras décadas del siglo XIX. Por otra parte Adame y Sánchez-Reciente fueron académicos correspondientes de varias Academias de distrito.

20 AHUS. Cátedras. Provisiones, libro 933. La secuencia, de acuerdo con las fechas de toma de posesión, fue la siguiente: 1830 (Sagrados Cánones), 1831 (Sagradas Escrituras y Decretales), 1832 (dos Cátedras de Instituciones Canónicas), 1834 (Teología Moral, dos Cátedras de Instituciones Médicas y tres Cátedras de Instituciones Civiles) y 1835 (una Cátedra de Instituciones Médicas, Anatomía, cuatro Cátedras de Instituciones Teológicas y Digesto Romano).

21 Real Decreto de 4 de agosto de 1836. Plan General de Instrucción Pública (Suplemento a la Gaceta de Madrid de 9 de agosto de 1836). Un análisis de este Decreto puede verse en PESET, José Luis. La enseñanza de la medicina en la España durante el siglo XIX. Minoría de Isabel II: regencias y gobierno provisional (1833-1843). Med. Esp., 63, 115-130 (1970), pp. 116-120.

22 Resulta significativo que la derogación se hiciese en una escueta Real Orden, que no se publicó en la Gaceta de Madrid, dirigida al Jefe Político de Burgos «por ser la materia de enseñanza pública privativa de las Cortes, hasta tanto la Dirección General establezca medidas para mejorar el Plan existente». Real 
texto legal tuvo un largo proceso de gestación ya que un año antes estaba pendiente de informe del Consejo Real, a pesar de lo cual se abrieron las Universidades en el curso 1835-1836 bajo la vieja normativa de 1824 «para que la juventud no sufra el más leve menoscabo así en su instrucción como en su carrera» ${ }^{23}$.

El inicio del curso 1836-1837 se vio marcado por varias circunstancias, unas generales y otras de carácter local. En primer lugar la situación política y bélica que sufría la Provincia de Sevilla y zonas limítrofes - «invadidas por los facciosos» en palabras del Rector - lo que dificultaba la apertura del curso ${ }^{24}$. Por otra parte, tras la derogación del «Plan General de Instrucción Pública» y la recuperación por parte de las Cortes y de la Dirección General de Instrucción Pública, ahora restablecida, de las competencias en estas materias, tuvo como consecuencia la publicación de un «Arreglo Provisional» aprobado por Real Orden de 29 de octubre de 1836 e inspirado por Manuel José Quintana, ahora presidente de la Dirección General ${ }^{25}$.

En lo tocante a la enseñanza de la medicina esta Real Orden contenía dos elementos que a corto plazo ponían en riesgo la continuación de los estudios médicos en las Universidades. En primer lugar éstas debían garantizar una calidad docente en la materia correspondiente al primer curso, disciplina ahora denominada «Anatomía descriptiva y general con nociones de fisiología». No podría iniciarse el curso si la Universidad en cuestión no disponía de los siguientes medios: Catedrático de la disciplina, Disector anatómico, Anfiteatro anatómico y cadáveres ${ }^{26}$. Por otra parte se establecían exigencias para la enseñanza Clínica como eran la de disponer de enfermos de «toda clase, edad y sexo» ${ }^{27}$. Es fácil percatarse que el «Arreglo Provisional» apuntaba hacia los cursos que contemplan enseñanzas que teóricamente, podían impartirse casi sin necesidad de libros. El discurso libresco podía fácilmente ser sustituido por el discurso del cadáver y el del enfermo; pero al mismo tiempo, para una enseñanza de estas características, se requería disponer de una infraestructura adecuada a estas estrategias docentes. La reforma de la enseñanza de la Anatomía y la de la Clínica se convertía en el elemento nuclear de la reforma médica.

Esta Real Orden fue oficialmente conocida por el Claustro de la Universidad de Sevilla ocho días después de su aparición en la Gaceta de Madrid y en él se solicitó a las distintas Facultades que presentaran un Dictamen sobre la situación de sus ense-

Orden de 4 de septiembre de 1836 . Decretos de la Reina Nuestra Señora Doña Isabel II. Dados en su Real
nombre por su augusta madre la Reina Gobernadora. Madrid, Imprenta Nacional, 1837, t. XXI, p. 372.
23 Real Decreto de 30 de septiembre de 1835 . Decretos de la Reina..., Madrid, Imprenta Real, 1836 ,
t. XX, pp. $443-444$.
24 Se acordó en Claustro general de 14 de noviembre de 1836 que la apertura se efectuara al día si-
guiente. AHUS. Libros de Claustros de la Real Universidad Literaria de Sevilla, libro 948, fols. $187 \mathrm{v}-188$.
25 Real Orden de 29 de octubre de 1836. Arreglo provisional de Estudios para el próximo año aca-
démico (Gaceta de Madrid de 6 de noviembre de 1836).
26 Ibidem, arts. 40 y 41 .
27 Ibidem, art. 43.
Asclepio-Vol. LIV-1-2002 
ñanzas ${ }^{28}$. La Comisión de Medicina, compuesta por Adame, Sánchez-Reciente, Velázquez y Campos, lo presentó en Claustro celebrado el 19 de noviembre señalando que se disponían de todos los medios requeridos: se iba a proceder al nombramiento de Catedrático para cubrir la vacante ocasionada por la reciente muerte de Velasco; se pensaba nombrar un Disector anatómico; se disponía de las instalaciones del Hospital Amor de Dios y cadáveres de esa procedencia; existían Catedráticos de Clínica; enfermos varones en este mismo hospital y de mujeres en el de la Sangre y se garantizaba que el futuro Disector anatómico ejecutaría autopsias clínicas. Algunos días después la Dirección General de Estudios requería del Rector que se le informase específicamente sobre la aplicación del «Arreglo» en medicina haciendo constar que debía pronunciarse «si para el estudio de la clínica hay el competente número de enfermos...debiendo el Catedrático de esa asignatura enseñar la anatomía patológica como parte esencial de la clínica» ${ }^{29}$.

Si en 1834 la Universidad de Sevilla declaró vacantes las cuatro Cátedras de Instituciones Médicas, no hizo lo mismo con la Cátedra de Clínica. En aquel momento acababa de morir José María Gavidia Espejo (1776-1833) que la regentaba por nombramiento claustral desde diciembre de 1824, fecha en que sustituyó a Velázquez Reyes. Este último la había servido durante el Trienio Liberal - fue al mismo tiempo Regidor Constitucional- y su conducta política le valió quedar cesante en 1824 y pasar a ocupar la sustitución de Gavidia entre 1825 y 1833. Tras la muerte de éste fue nombrado nuevamente Catedrático por el Claustro y propuso para sustituirle a su sobrino Francisco Porrúa Velázquez. Porrúa era igualmente liberal y su posición política — siendo aun estudiante - fue calificada de «dudosa» en 1824. La quebrantada salud de Velázquez le llevó a renunciar a la Cátedra en 1836, que pasó a manos de su sobrino no sin gran oposición por parte del sector más conservador de la Facultad que intentaron bloquear este nombramiento en el Claustro proponiendo a Adame ${ }^{30}$.

La enseñanza Clínica, desde su implantación, venía haciéndose en el Hospital Amor de Dios al que acudían los Catedráticos y los alumnos a enseñar y aprender a la cabecera del enfermo. El costo de estas camas destinadas a la enseñanza clínica - 12 en 1820 - atendidas por Catedráticos y no por médicos del hospital, parece ser que era superior al resto de las camas hospitalarias, por lo menos en lo que a gastos tera-

28 AHUS. Libros de Claustros..., libro 948, fols. 187v-188.

29 AHUS. Libros de Claustros..., libro 948, fols. 191-192v. Reales Órdenes, Pragmáticas, Cédulas y Provisiones dirigidas a la Universidad de Sevilla, libro 972, fol. 119 [Provisión de la Dirección General de Estudios al Rector de 30 de noviembre de 1836].

30 AHUS. Libros de Claustros..., libro 948, fols 197v-198v. Aparte de Porrúa presentó su candidatura Manuel Pérez Martínez que era el sustituto de Adame y por otra parte los tres Catedráticos, Campos, Sánchez-Reciente y Adame, propusieron a este último así como que se proveyera en sustitución la Cátedra de Instituciones de $4^{\circ}$ curso que se desocupaba. Es posible que la operación, de haber resultado exitosa, llevara aparejado más tarde el nombramiento de Pérez Martínez para esta Cátedra y el desplazamiento del liberal Porrúa. 
péuticos se refiere, fenómeno que desequilibraba el presupuesto. Así ocurrió en 1832 en que el administrador del Hospital Diego Martínez de Mora lo expuso al Claustro solicitando se tomasen medidas económicas que permitieran «conciliar con la imposibilidad del Hospital, la continuación de la enseñanza de Clínica en el caso de que se resolviese que permanezca en el mismo» ${ }^{31}$.

Es evidente que en 1836 no se reunían ninguna de las condiciones previstas en el «Arreglo», pero ello forzó a la puesta en marcha de un proceso tendente a mejorar la enseñanza. En primer lugar cubrir la vacante de Velasco. Presentaron sus candidaturas ante el Claustro Joaquín Palacios Rodríguez (1815-1887) y Fernando Santos de Castro (1809-1890) y en el celebrado el día 26 de noviembre nombró como Catedrático de Anatomía al primero. Por otra parte se procedió al nombramiento de Disector anatómico en la persona del cirujano y estudiante de $3^{\circ}$ curso de medicina Antonio García Portillo y como Catedrático sustituto de Anatomía continuó Doy Burgos ${ }^{32}$. En lo referente a la enseñanza Clínica se nombró, ya lo hemos dicho, a Porrúa y pronto el Hospital de la Sangre - ahora denominado Hospital Central— se convertiría en el espacio destinado a este fin $^{33}$.

No parece que preocupase demasiado en Sevilla - a diferencia de lo que ocurrió en Valencia ${ }^{34}$ - el proyecto de reforma de la enseñanza médica elaborado por la Dirección General y que no llegó a ser aprobado por las Cortes en 1837. Nuevamente se apuntaba hacia una reducción del número de Facultades de Medicina al permitirse exclusivamente matriculaciones del primer curso en las Universidades de Valladolid, Santiago y Zaragoza, lo que a muy corto plazo hubiera significado el cierre de las restantes Facultades, conservándose, eso sí, los Colegios de Medicina y Cirugía por los que Quintana, muy razonablemente, sentía predilección ${ }^{35}$.

En 1839 los dos Catedráticos incorporados por acuerdo del Claustro tres años antes -Palacios en Anatomía y Porrúa en Clínica - fueron consolidando su posición hasta tal punto que solicitaron al Rector que ambas Cátedras fueran cubiertas en vir-

31 AHUS. Libros de Claustros..., libro 948, fols. 139-139v.

32 AHUS. Libros de Claustros..., libro 948, fols. 195-195v, 199v y 202

33 Hay que tener en cuenta que en 1837 desapareció el Hospital del Amor de Dios integrándose en el nuevo Hospital Central ubicado en el edificio del Hospital de las Cinco Llagas (también llamado Hospital de la Sangre). En 1843 se usaba la Clínica de medicina de este Hospital para la enseñanza y se solicitaba al Gobierno por el recién creado Colegio de Prácticos la extensión a la Clínica quirúrgica y a la de Obstetricia. El número total de camas hospitalarias que se consideraban necesarias para la docencia eran: entre 24 y 30 de las clínicas médica y quirúrgica respectivamente y 12 de la clínica de partos y enfermedades de las mujeres. AHUS. Colegio de Prácticos de Medicina de Sevilla. Juntas y Grados, libro 955 [Presupuesto para el Colegio de Prácticos, 24 de diciembre de 1843].

34 García BAllester, L.; BAlaguer PerigüEl, E. (1969) «Protestas por la supresión de la Cátedra de Anatomía de Valencia (1837)», en III Congreso Nacional de Historia de la Medicina, Actas, Valencia, vol. II, pp. 385-389.

35 PeSET, J. L. (1970), p. 120 
tud de oposición, naturalmente bajo la misma normativa con la que en 1834 las habían conseguido sus compañeros de Claustro. La Real Orden de 1 de julio de 1835, a la que ya hemos hecho alusión, aun vigente en aquella fecha, suspendió cualquier provisión de Cátedras hasta que el Gobierno elaborase una reforma de las enseñanzas y como tal reforma no se había producido su petición fue denegada ${ }^{36}$.

\section{III) EL IMPACTO DE LA REVOLUCIÓN DE SEPTIEMBRE EN LA ENSEÑANZA DE LA MEDI- CINA EN SEVILLA}

La noche del 15 de septiembre de 1840 se produjo en Sevilla un pronunciamiento con la constitución de una Junta Directiva de Gobierno de la Provincia presidida por Manuel Bayo Sologuren, Alcalde $2^{\circ}$ Constitucional, que de inmediato determinó destituciones políticas y el restablecimiento de la Milicia Nacional. Una de sus primeras decisiones políticas fue la de exigir a los empleados y funcionarios públicos que en el plazo de 24 horas presentaran su dimisión espontánea y voluntariamente si no reconocían la autoridad de la misma ${ }^{37}$; hecho que se extendió poco más tarde a los funcionarios de clases pasivas ${ }^{38}$. La Universidad no quedó fuera de esta exigencia de reconocimiento y acatamiento político. El recién nombrado Jefe Político de la Provincia, Joaquín Garrido, se dirigió expresamente al Claustro Universitario exigiéndole a sus miembros un pronunciamiento en tal sentido y reunido el día 23 de septiembre, los cinco claustrales de medicina firmaron su adhesión ${ }^{39}$. Sin embargo tres días después se procedía a la separación temporal de cinco Catedráticos, que interesaba a la casi totalidad de la cúpula universitaria: el Rector Nicolás Maestre y Tous de Monsalve (1766-1841); Juan Nepomuceno Escudero, Catedrático de Oratoria y Depositario; Joaquín Sánchez-Reciente Díaz, Secretario-Contador; los Catedráticos de Leyes Antonio María de Rodas y José Rivero de la Herranz y el Catedrático de Instituciones Canónicas Ramón de Beas y Dutari. El oficio de la Junta revolucionaria al Claustro en el que se comunicaba tal decisión establecía con claridad que las vacantes producidas por estas separaciones debían ser cubiertas «por personas que además de su aptitud científica sean amantes de la libertad y del sistema nacional restablecido». Es significativo que el que era Síndico-Fiscal desde 1837 y Catedrático de Clínica, el liberal Francisco Porrúa, fuera el único de la cúpula no separado ${ }^{40}$. No satisfecha la Junta revolucionaria con estas medidas, se obligó a que todas las autoridades civiles, militares, eclesiásticas y los funcionarios públicos se pronunciaran explícitamente

\footnotetext{
36 AHUS. Índice de carreras, libro 833, fol. 213; Reales Órdenes..., libro 972, fol. 193.

37 Boletín Oficial de la Provincia de Sevilla, $\mathrm{n}^{\circ} 1610$ de 16 de septiembre de 1840.

38 Ibidem, $\mathrm{n}^{\circ} 1613$ de 22 de septiembre de 1840.

39 AHUS. Libros de Claustros..., libro 948, fols. 228v-229.

40 AHUS. Libros de Claustros..., libro 948, fols. 230-230v.
} 
bajo la siguiente fórmula: «Reconozco la autoridad popular de la Junta Superior Directiva de esta Provincia y me adhiero a los principios políticos que representa el pronunciamiento Nacional que la establece» ${ }^{41}$.

Tenemos constancia de que tanto Beas como Escudero fueron más tarde repuestos en sus Cátedras; pero no es este el caso de Sánchez-Reciente, personaje muy significado políticamente desde el Trienio y que ahora sufrirá en sus propias carnes la represión política. En el mismo Claustro que se le separaba de la Cátedra se nombraba para sustituirle a Fernando Vida Pérez (1799-d.1873). Puede resultar significativo que todos los logros académicos y profesionales de este ecijano estén casi siempre relacionados con el entorno liberal, aprovechando las posibilidades que éste le ofrecía. Su vinculación a la Universidad de Sevilla data de 1836 en que fue nombrado Catedrático sustituto de Matemáticas; dos años más tarde fracasó en su intento de ser nombrado Catedrático sustituto de Filosofía y poco después de ser nombrado Catedrático sustituto de Instituciones Médicas de $3^{\circ}$ año, consiguió que el Claustro, por 26 votos de sus miembros, le nombrase Catedrático Interino de la misma disciplina, lo que llevaba aparejado «honores y sueldo de propietario» ${ }^{42}$. Esta misma categoría sería lograda gracias a una Orden de Espartero, un año más tarde, por Francisco Porrúa ${ }^{43}$. Por el contrario Palacios, el Catedrático sustituto de Anatomía, permaneció inmodificado en su status académico.

Sánchez-Reciente desaparece casi por completo del panorama médico sevillano tras su separación académica ${ }^{44}$ y emergerá en 1844 en el que intentó ser repuesto en su Cátedra y en el cargo de Contador. Por una parte el Ministro Pidal decidió que tal separación no constase como nota negativa en su carrera, pero por otra dejará que sea el propio Claustro de la Universidad el que decida sobre su readmisión. La decisión se tomó en el Claustro celebrado el 21 de junio de 1844: efectuada una votación y por mayoría de sus miembros se aprobó la no readmisión del Catedrático separado ${ }^{45}$.

Desde 1835 uno de los problemas que más ocuparon a la Junta Superior Gubernativa de Medicina y Cirugía fue el de la regulación de la unión de la enseñanza de la medicina y la profesión médica. En tal sentido se adoptaron medidas políticas tendentes a hacer posible una profesión médica unificada facilitando que los cirujanos

41 Boletín Oficial de la Provincia de Sevilla, $\mathrm{n}^{\circ} 1615$ de 25 de septiembre de 1840; AHUS. Libros de Claustros..., 948, fols. 234v-235.

42 AHUS. Libros de Claustros..., libro 948, fols. 215v, 230v y 253v.

43 AHUS. Índice de carreras, libro 833, fol. 44 [Orden del Regente dada en Madrid el 17 de mayo de 1841]. La categoría de Catedrático Interino no estaba recogida como tal en la Real Orden de 14 de octubre de 1824, aunque sí implícitamente pues debía tratarse del tipo de sustituto contemplado en su art. 217.

44 Archivo de la Real Academia de Medicina y Cirugía de Sevilla (ARAMCS). Libro de Actas (1841-1844). Tanto Sánchez-Reciente como su padre, que también era Académico desde 1831, dejan de asistir a las sesiones de la Academia.

45 AHUS. Índice de carreras, libro 843, fols. 555-556; Libros de Claustros..., libro 948, fols. 278 y $280-280 \mathrm{v}$ 
pudieran licenciarse en medicina y los estudiantes de medicina y los médicos pudiesen optar a la Licenciatura en Cirugía, todo ello a través de una regulación de los correspondientes estudios complementarios ${ }^{46}$. Como es obvio la Universidad de Sevilla sólo impartía enseñanza para la formación de médicos; pues bien, uno de los logros de la Junta revolucionaria fue la implantación de estudios quirúrgicos que hicieran posible la formación integral médico-quirúrgica en el seno de la misma.

El proceso se inició como consecuencia de la toma en consideración de una petición en tal sentido elevada a la Junta por «varios [y desconocidos, apostillamos nosotros] individuos de la clase de Clínica de esta ciudad». Fueron los peticionarios Andrés Calero de las Navas, que aun no había alcanzado en Grado de Bachiller en Medicina; Policarpo Macías Flores, Bachiller en Medicina aquel mismo año; Juan D. A. Galán y Alfonso Romero Marta. Resulta significativo el algo grado de receptividad de la Junta a una iniciativa que casi la podríamos calificar de «popular». En su escrito de petición de 2 de octubre de 1840 —en plena efervescencia revolucionaria- se solicitaba la creación de una Cátedra de Cirugía y se fundamentaba sobre conceptos de territorialidad, bondad de los Hospitales sevillanos y disponibilidad de cadáveres. Varios días después la Junta mandataba a la Universidad la implantación de estos estudios en aquel mismo curso académico ${ }^{47}$.

El informe elaborado por el Claustro de la Facultad de Medicina señalaba el atraso en que había quedado la medicina a partir de 1824 —el referente temporal es significativo- y proponía un plan que consistía en la dotación de dos Cátedras cuyas enseñanzas se impartirían en los cursos $5^{\circ}$ y $6^{\circ}$, coincidiendo con la enseñanza clínica propia de la Licenciatura de Medicina. Estos cursos serían: «Nosografía y Patología especial externa. Vendajes y Operaciones» con asistencia a la Clínica Quirúrgica en el Hospital y «Obstetricia, enfermedades de las mujeres y niños y sifilíticas» con asistencia tanto a la Clínica Quirúrgica como a la Médica. En cierta medida los cursos propuestos eran muy parecidos a los que desde 1836 se exigían a los médicos que deseaban optar a la Licenciatura en Cirugía, con una notable excepción, que en éstos se contemplaban nuevos estudios de Anatomía cosa que no ocurría en la propuesta del Claustro de Medicina. El informe a que estamos haciendo referencia incluía también una propuesta de posible profesorado para ambos $\operatorname{cursos}^{48}$. A esta primera relación de candidatos se sumaron más tarde algunos otros y en votación celebrada en el Claustro de 18 de noviembre se eligieron a Antonio Marsella Sierra (1808-1874) para ocupar la primera de estas Cátedras y a Joaquín Palacios Soto-Sánchez (c.1792-

\footnotetext{
46 Peset, J. L. (1970), pp. 116-117.

47 AHUS. Libros de Claustros..., libro 948, fols. 241-241v.

48 AHUS. Libros de Claustros..., libro 948, fols. 245-247v.
} 
d.1852), para la segunda ${ }^{49}$. Ambos habían sido alumnos del Colegio de Medicina y Cirugía de Cádiz.

El éxito de estos cursos — sobretodo el impartido por Marsella-, al que no fueron ajenos los intereses profesionales, fue inmediato con una notable matriculación de médicos y alumnos en su periodo clínico ${ }^{50}$. Baste señalar que en el curso 18401841 se matricularon 70 alumnos y en el siguiente 31 en primero y 52 en segundo; entre este alumnado se encontraban Doctores en Medicina como Antonio Rivera Ramos (1815-1887) e incluso Catedráticos como Porrúa, Vida o Sánchez-Reciente ${ }^{51}$, este último sólo cursó el primer año por las circunstancias ya mencionadas ${ }^{52}$. A pesar de este éxito la situación de Marsella era de gran precariedad al estar impartiendo una docencia cuya validez estaba siendo seriamente cuestionada desde el poder central y tal vez por ello intentase consolidar su posición aspirando a la categoría de Catedrático sustituto ${ }^{53}$.

Casi de inmediato se fue consolidando un proceso de centralización resultado de una política en materia de enseñanza cada vez más intencionalmente dirigida por Espartero y sus gobiernos. En un primer momento se cuestionaron todas las iniciativas que en el campo de la enseñanza habían tomado las Juntas Provinciales argumentando que muchos establecimientos se mantenían al margen de la ley: estudios privados, conversión de antiguos Colegios en Universidades, etc. La situación de la enseñanza de la Cirugía en Sevilla presentaba, cuando menos, serias dudas en relación con la validez legal de estos estudios. Para clarificar esta situación el Claustro de Medicina elevó consulta a la Dirección General y ésta fue contundente en su respuesta: «que los estudios quirúrgicos hechos por los escolares en ese establecimiento podrán ser validados en los Colegios [de Cirugía] mediante un examen en ellos siempre que por estos resulten aprobados» ${ }^{54}$. Pocos días después se llegó más lejos al

49 En realidad se efectuaron dos votaciones diferenciadas para cada una de las Cátedras. Para la primera Marsella obtuvo 16 votos y Joaquín Palacios 8 votos; para la segunda Janer [posiblemente Juan José Sánchez Janer] obtuvo 16 votos y Palacios 5 votos. Al final se optó por nombrar a los dos candidatos que habían obtenidos más votos para la primera Cátedra por renuncia de Janer. AHUS. Libros de Claustros..., libro 948, fols. 250-251v.

50 Sobre la creación de esta Cátedra y su ocupación por Marsella disponíamos del testimonio de LASSO DE LA VEGA, J. (1875) «Apuntes biográficos», en Sesión Literaria en honor del Dr. Antonio Marsella Sierra, Sevilla, Tarascó, p. 9. Para una aproximación al problema basada en este testimonio $c f$. DOMíNGUEZ RODIÑO, E. (1983), «Los cirujanos románticos operaban antes de existir la anestesia», Chequeo, $1, \mathrm{n}^{\circ}$ 0, 34-42, pp. 38-39.

51 AHUS. Matrículas (1773-1845), libro 513, s.f.

52 AHUS. Exámenes. Listas de exámenes (1795-1845), libro 523

53 AHUS. Índice de carreras, libro 824, fols. 177-179v; Orden de 31 de agosto de 1841 (Gaceta de Madrid de 5 de septiembre de 1841). Marsella se acogió a la disposición $5^{\text {a }}$ que regulaba el modo de alcanzar la condición de sustituto de Cátedra, nombramiento efectuado a partir de ese momento por el Gobierno en lugar del Claustro de la propia Universidad.

54 AHUS. Reales Órdenes..., libro 972, fol. 290 [Provisión de 25 de junio de 1842]. 
determinar el Ministro de la Gobernación, atendiendo un Dictamen de la Dirección General, la supresión de estos estudios en la Universidad de Sevilla, dictamen que se apoyó sustancialmente en la carencia de formación anatómica. El texto de la Orden trasladada al Rector es especialmente significativo: «S. A. el Regente del Reino conformándose con el dictamen de esa Dirección General se ha servido mandar que se supriman las dos Cátedras de Cirugía abiertas en la Universidad Literaria de Sevilla por la Junta de Gobierno de 1840, en atención a carecer aquel establecimiento de los medios necesarios para el estudio de la expresada Facultad y a ser tan incompletas las dos asignaturas de que queda hecho mérito que ni siquiera se ha contado al establecerlas con la importante Cátedra de Anatomía, cuyos conocimientos por absolutamente precisos para la parte operatoria, en manera ninguna pueden dispensarse al que haya de ejercer en profesión de cirujano» ${ }^{55}$. No debió hacer mucho caso la Universidad a este mandato cuando casi un año después el propio Regente la obligaba a ejecutar la orden de supresión ${ }^{56}$.

IV) LAS REFORMAS DE 1843-1845: DE LA CREACIÓN DEL COLEGIO DE «PRÁCTICOS EN EL ARTE DE CURAR» A LA SUPRESIÓN DE LOS ESTUDIOS MÉDICOS EN SEVILLA

El Real Decreto de 10 de octubre de 1843 del Gobierno provisional -firmado por el Ministro Fermín Caballero e inspirado por el médico-legista Pedro Mata - fue el comienzo de un proceso de profundas reformas de la enseñanza de la medicina con notables repercusiones sobre la profesión médica. En él se unificaba definitivamente la enseñanza de la medicina y la cirugía, desaparecía la enseñanza de la primera en las Universidades, se transformaban en Facultades Universitarias los antiguos Colegios de Cirugía de Madrid y Barcelona y se creaban los Colegios de «Prácticos en el Arte de Curar», para la formación de un profesional sanitario capacitado exclusivamente para enfrentarse a problemas médicos elementales, cirugía menor y asistencia a partos. La aplicación de este Real Decreto tuvo un severo impacto en la Universidad Literaria de Sevilla: desapareció su Facultad de Medicina y se creó un Colegio de Prácticos ${ }^{57}$.

El profesorado de esta nueva institución docente universitaria fue nombrado por el Gobierno reclutándolo básicamente entre los que venían desarrollando su docencia en la Facultad suprimida. La situación en aquel momento era la siguiente: se disponía de dos Catedráticos propietarios (Adame y Campos), dos Catedráticos Interinos con

\footnotetext{
55 AHUS. Reales Órdenes..., libro 972, fol. 308 [Orden de 20 de agosto de 1842].

56 AHUS. Reales Órdenes..., libro 972, fol. 349 [Orden de 18 de mayo de 1843].

57 Real Decreto de 10 de octubre de 1843. Estableciendo un nuevo plan de estudios médicos (Suplemento a la Gaceta de Madrid de 11 de octubre de 1843), art. 1 (supresión de la Facultad de Medicina de la Universidad Literaria de Sevilla); art. 3 (creación del Colegio de Prácticos en Sevilla) y art. 30 (enseñanza).
} 
honores y sueldo de propietario (Vida y Porrúa) y un Catedrático sustituto (Palacios Rodríguez). El Plan de Estudios del Colegio de Prácticos establecía la existencia de cinco asignaturas cada una a cargo de un Catedrático: 1) Anatomía descriptiva y fisiología; 2) Higiene, terapéutica, materia médica y arte de recetar; 3) Anatomía, patología, clínica quirúrgica y vendajes; 4) Patología médica, obstetricia y clínica de partos y 5) Patología general, medicina legal y clínica médica. Para la ocupación de estas Cátedras en Sevilla el Gobierno provisional nombró como Catedráticos propietarios a Palacios ( $1^{\mathrm{a}}$ Cátedra), Vida ( $2^{\mathrm{a}}$ Cátedra), Campos $\left(4^{\mathrm{a}}\right.$ Cátedra) y Adame ( $5^{\mathrm{a}}$ Cátedra). Para la tercera Cátedra — esencialmente quirúrgica — nombró a Juan Ceballos Gómez (1817-1874) que presentaba como méritos, a los ojos del Gobierno, ser «opositor» a Cátedras, Académico por oposición y publicista ${ }^{58}$. Este gaditano había estudiado en el Colegio de Medicina y Cirugía de Cádiz, alcanzando el Grado de Doctor en $1841^{59}$. Quedarán fuera de esta reordenación Porrúa- sus enseñanzas clínicas se habían impartido en el periodo de Licenciatura- y Marsella, a pesar de tener un perfil bastante similar al de Ceballos y acreditar una experiencia docente exitosa en 1840 y $1841^{60}$.

En la nueva ordenación universitaria desaparecieron los sustitutos de Cátedra y fueron remplazados por una nueva figura, los Profesores Agregados, con funciones algo más extensas que las que aquéllos tuvieron ${ }^{61}$. En Sevilla, de acuerdo con la normativa vigente, se nombraron a los siguientes: Antonio Rivera Ramos, para la $2^{\mathrm{a}}$ Cátedra; Joaquín Palacios Soto-Sánchez, para la $3^{\mathrm{a}}$ y parte de la $4^{\mathrm{a}}$ (Obstetricia) Joaquín Ballesteros Sousa, para el resto de la $4^{\mathrm{a}}$ y la $5^{\mathrm{a}}$. Por otra parte se nombró como Director de los trabajos anatómicos a Vicente Domínguez Daza (1816-d.1902) ${ }^{62}$. Un

\footnotetext{
58 Ministerio de la Gobernación de la Península. Negociado $n^{\circ}$ 15. Circular (Gaceta de Madrid de 7 de noviembre de 1843).

59 Sobre Juan Ceballos $c f$. OROZCo ACUAVIVA, A. (1981), Bibliografía médico-científica gaditana. Ensayo bio-bibliográfico médico, científico y técnico de Cádiz y su provincia. Cádiz, Obra Cultural «Casino Gaditano», pp. 201-202.

60 Marsella también había estudiado en el Colegio de Medicina y Cirugía de Cádiz y era Académico de la Real Academia de Medicina y Cirugía de Sevilla desde 1842. Posiblemente un factor fundamental en esta decisión del Gobierno en favor de Ceballos fuera el hecho de ser desde 1839 Director de la Revista Médica Gaditana.

61 Real Decreto de 10 de octubre de 1843 (Suplemento a la Gaceta de Madrid de 11 de octubre de $1843)$, arts. 15 y 38 .

62 AHUS. Colegio de Prácticos de Medicina de Sevilla. Juntas y Grados, libro 955 [Sesión de 10 de enero de 1844 en la que se elaboró la lista de candidatos y eran estos: Francisco Porrúa Velázquez, Joaquín Ballesteros Sousa, Joaquín Palacios Soto-Sánchez, Vicente Domínguez Daza, Manuel Pérez Martínez, Francisco de Paula Gómez Montero de Espinosa (n. 1816), Antonio Colom Osorio (n. c. 1807) y Narciso García Romero. En sesión de 8 de febrero renunciaron estos dos últimos al no aceptar un nombramiento gubernamental. En sesión de 29 de febrero se dio cuenta del nombramiento de los tres Agregados y del Director de trabajos anatómicos y en la de 1 de marzo en la que se dio posesión a los tres Agregados]
} 
año después se constituyó la «Escuela Práctica» que quedaría a cargo de estos profesores Agregados ${ }^{63}$. Como Director y Sub-director se nombraron respectivamente a Adame y Ceballos ${ }^{64}$.

Pronto esta estructura del profesorado empezó a mostrar signos de inestabilidad. Ceballos se incorporó en junio de 1844 a la restaurada Facultad de Ciencias Médicas de Cádiz y para cubrir esta baja fue nombrado por el Gobierno León SánchezQuintanar y Sánchez Nieto (1801-1877) en virtud de sus «méritos y servicios» ${ }^{65}$. La jubilación de Adame en aquella misma fecha se resolvió, igualmente por el Gobierno, con un nuevo nombramiento, en este caso el de Juan Nepomuceno Torres Yáñez, un Catedrático cesante en la Universidad de Granada como consecuencia de la reforma y que no llegó a incorporarse al Colegio de Sevilla ${ }^{66}$. Esta situación se resolvió por el Gobierno dejando sin efecto la jubilación de Adame ${ }^{67}$. Domínguez Daza, como Ceballos, pasó a Cádiz con igual cargo cuatro meses después de su nombramiento, siendo sustituido por José Moreno Fernández (1823-1899) durante el curso académico $1843-1844$ y a partir se enero de 1845 asumió estas responsabilidades el propio Catedrático de la asignatura ${ }^{68}$. Lo más significativo de todos estos movimientos fue la incorporación de Sánchez-Quintanar. De talante liberal y progresista, se había formado en el Real Colegio de San Carlos de Madrid y su corta estancia en Sevilla fue un revulsivo en el modesto panorama docente de la ciudad. Organizó las enseñanzas de las materias quirúrgicas correspondientes a la $3^{\text {a }}$ Cátedra y como manifestación de su mentalidad anatomo-clínica promocionó la autopsia y los estudios anatomo-patológicos desde la enseñanza quirúrgica ${ }^{69}$.

El final de la enseñanza de la medicina en la Universidad de Sevilla se aproximaba. Por Real Decreto de 17 de septiembre de 1845 se suprimían los Colegios de Prácticos y se establecían cinco Facultades de Medicina en todo el territorio: Madrid y Barcelona, que ya funcionaban desde 1843; Cádiz, que la recuperó en 1844 y Santiago y Valencia, que transformaron sus Colegios de Prácticos ${ }^{70}$. A partir de este mo-

63 La Escuela Práctica estaba prevista en los arts. 18 y 41 del Real Decreto 10 de octubre de 1843 y en ella los profesores podían impartir cursos públicos o privados. AHUS. Colegio de Prácticos de Medicina de Sevilla. Juntas y Grados, libro 955 [Sesión de 29 de noviembre de 1844].

64 AHUS. Reales Órdenes..., libro 972, fol. 414.

65 Sobre la etapa sevillana de Sánchez-Quintanar $c f$. Micó NAVARro, J. A. (1987), León SánchezQuintanar (1801-1877). Vida, obra y biblioteca, Valencia, Tesis Doctoral inédita, pp. 35-38; AHUS. Cátedras. Provisiones, libro 933, fols. 46-47.

66 AHUS. Colegio de Prácticos de Medicina de Sevilla. Juntas y Grados, libro 955; Índice de carreras, libro 781 , fol. 468 .

67 AHUS. Colegio de Prácticos de Medicina de Sevilla. Juntas y Grados, libro 955 [Sesión de 1 de diciembre de 1844].

68 AHUS. Colegio de Prácticos de Medicina de Sevilla. Juntas y Grados, libro 955. [Sesiones de 9 de enero y 30 de agosto de 1845].

69 Micó NAVARRO, J. A. (1987), pp. 36-37.

70 Real Decreto de 17 de septiembre de 1845 (Gaceta de Madrid de 25 de septiembre de 1845), art. 75. 
mento la Universidad Literaria de Sevilla tendrá su Facultad de Medicina en la ciudad de Cádiz. Esta supresión de los estudios médicos en Sevilla fue vista desde una óptica diferente: fue un acontecimiento totalmente injustificado para José Velázquez Sánchez porque «cortó la carrera de no pocos aventajados alumnos, faltos de medios para subsistir en aquella localidad [Cádiz], a la vez que privó a la enseñanza de profesores de nota» ${ }^{71}$. No tan injustificada veía esta decisión Federico Rubio Galí (18271902) en aquel momento estudiante de medicina en Cádiz, centro docente al que llegaron para terminar sus estudios buena parte de los estudiantes sevillanos que «A la verdad, los Bachilleres [en Medicina] sevillanos de entonces venían muy mal educados social y científicamente. El mayor número vestía calesera y faja, capa corta y calañé; escupían por el colmillo y no entendían que hubiese honra ni dignidad personal sin ser un jaque, en toda la extensión de la palabra. Científicamente, los aplicados traían una solera extraña de Boerhaave y Broussais que ellos habían compuesto de los dos bandos de maestros que explicaban en la Universidad $\gg^{72}$.

Con la clausura del Colegio de Prácticos todo el profesorado quedó cesante y su posterior destino estuvo íntimamente vinculado a las características personales y profesionales de cada uno de ellos. Manuel Campos Vallejo tenía 65 años y falleció tres años más tarde. Juan Nepomuceno Torres Yáñez sería integrado por el Gobierno en el Escalafón Nacional en 1845 formando parte del Claustro de la Universidad de Valencia como Catedrático de Medicina Legal e Higiene Pública y en 1854 regresaría a la Universidad de Granada en la que llegó a ser Rector en 185773. León Sánchez-Quintanar fue nombrado un año más tarde Catedrático de Historia Natural Médica en la Universidad de Santiago, para casi de inmediato pasar a la de Valencia ocupando la Cátedra de Patología Quirúrgica ${ }^{74}$.

Muy significativos fueron los casos de los sevillanos Palacios y Vida que se acogieron a las posibilidades que les ofrecía el mismo Real Decreto por el que cesaron en la enseñanza universitaria. El primero fue acoplado de inmediato al Instituto de $2^{\mathrm{a}}$ enseñanza de Sevilla como Catedrático Interino de Elementos de Geografía, obtuvo el Grado de Licenciado en Letras en 1846 y este mismo año fue nombrado, por el Rector, Regente de $1^{\text {a }}$ clase, llegando a ser durante 26 años el Director del mencionado Instituto. Durante estos años continuó su labor publicista - había sido promotor del escaso y efímero periodismo médico publicado en Sevilla a comienzos de la dé-

71 VelázQuez SÁNCHEZ, F. (1872), Anales de Sevilla de 1800 a 1850. Sevilla, Imprenta y Librería de Hijos de Fé, p. 629.

72 RuBIO, F. (1888), «Recuerdo necrológico del Instituto de Terapéutica Operatoria», en Ariza Espejo, R. Estudios Médicos. Madrid, Imprenta y Librería de Enrique Teodoro, 1888, vol. III, p. 471.

73 Proyecto de Escalafón General de Catedráticos propietarios de actual servicio en las Universidades del Reino [1845]. Boletín Oficial de Instrucción Pública, nº 10, 1846; RAMALlo ORTIZ, J. A. (1976), Catálogo de profesores de la Universidad de Granada (1845-1935), Granada, Imprenta Román, p. 137.

74 Micó NAvarro, J. A. (1987), p. 39. 
cada de los cuarenta - preocupándose de elaborar diversos manuales de Geografía e Historia destinados a sus estudiantes del Instituto ${ }^{75}$. Fernando Vida encontró una salida muy similar a la de Palacios. Fue nombrado en 1845 Catedrático sustituto de Mitología y principios de Historia en el Instituto sevillano y un año después alcanzó el Grado de Licenciado en Ciencias y Regente de $2^{\circ}$ clase de la Cátedra de Historia y en 1847 Catedrático propietario, permaneciendo en esta situación hasta 1850 en que se suprimió esta Cátedra y fue acumulada a la de Geografía que regentaba Palacios ${ }^{76}$. Tras dos años de cesantía, por Real Orden de 12 de mayo de 1852, fue nombrado Catedrático de Obstetricia de la Universidad de Valencia y dos años más tarde pasó a ocupar la Cátedra de Patología general, terapéutica, materia médica y arte de recetar en la misma Universidad. Aprovechando sus buenas relaciones políticas logró ascensos de forma irregular y llegó a ser Decano de la Facultad de Medicina y Vice-Rector de la Universidad. En lo que no parece que ascendiera fue en su cualificación académica e intelectual ${ }^{77}$.

La salida de los tres Agregados se caracterizó por su total desvinculación con la enseñanza. Palacios Soto-Sánchez intentó seguir el mismo camino que su hijo y solicitó al Rector que se le nombrara para ocupar una Cátedra vacante de Filosofía en la Universidad y si esto no fuera posible que se le acoplara en alguna de $2^{\mathrm{a}}$ enseñanza (Latín, Retórica y Poética, Geografía, Mitología e Historia, Nociones de Historia Natural). En su opinión era apto para cualquier materia. No parece que alcanzase su objetivo $^{78}$. Rivera Ramos y Ballesteros Sousa se dedicaron a su ejercicio profesional alcanzando ambos, pero sobretodo el primero, un alto grado de prestigio y formaron parte de la elite médica sevillana. Cuando en 1868, por Decreto de la Junta Revolucionaria a iniciativa de Federico Rubio que se amparó en el principio de liberalización de la enseñanza, se cree la Escuela Libre de Medicina y Cirugía de Sevilla, se recuperarán la casi totalidad de los enseñantes supervivientes de la década de los cuarenta: Palacios Rodríguez, Ballesteros Sousa, Moreno Fernández, Marsella Sierra y Rivera Ramos. Estos tres últimos llegaron a ser piezas fundamentales en los primeros 30 años de vida de la Escuela ${ }^{79}$.

75 AHUS. Expedientes académicos, leg. 140-15-34; MÉNDEZ BEJARANO, Mario. Diccionario de Escritores, Maestros y Oradores naturales de Sevilla y su actual provincia. Sevilla, Tipografía Gironés, 1923, vol. II, p.192.

76 AHUS. Expedientes académicos, leg. 186-7-57.

77 Archivo de la Universidad de Valencia (AUV). Foll. 12/33; L.179 (189) L.425 (72). Todos cuantos se han acercado al problema de la enseñanza en la Facultad de Medicina de Valencia reconocen la escasa actividad de Vida. Sobre esto $c f$. LÓPEZ PIÑERO, José Ma . (1992), «Los saberes médicos y su enseñanza», en López Piñero, J. Mª (Dir), Historia de la Medicina Valenciana, Valencia, Vincent García Editores S.A., vol. III, p. 35.

78 AHUS. Índice de carreras, libro 831, fols. 397 y 402.

79 Sobre la Escuela Libre de Medicina y Cirugía de Sevilla $c f$. CARRILlO, J. L. (1990), «Medicina y enseñanza de la medicina en Sevilla (1868-1883): continuidad y cambio», Dynamis, 10, pp. 163-192. 